\title{
Primera prueba del Concurso de Nosografía Médica
}

\author{
POR EL DOCTOR
}

CARLOS MONGE

Señores Cátedráticos:

Señores:

Quiere la ley que el opositor a un concurso exponga en esta prueba los fundamentos de su programa.

Para proceder con orden, voy a ocuparme en primer lugar de cual debe ser el espíritu del profesorado; en seguida de las bases y finalidades del curso de Patología Interna; luego del criterio con que debe estudiarse ésta, y finalmente del método pedagógico que conviene a su enseñanza.

\section{I.-El espíritu del profesorado}

Porque sé que há de enseñarse con el ejemplo es que mi primera palabra ha de ser un homenaje a la memoria de los que lo dieron, dejándonos la herencia de su labor constructiva en el profesorado nacional. Bien vale, pues, la pena detenerse un momento, volver la vista al pasado sobre cuyos hombros contemplamos el porvenir, y vivir un instante de recogimiento, no para llorar a los muertos, sino para llenarnos el alma del orgullo silencioso, sugerente y evocador, que colma el espíritu cuando se contempla la obra que llevaron a cabo los que fueron-ayer no más-valores máximos educadores en la centenaria casa de San Fernando.

Porque sé que ha de enseñarse con el ejemplo es que quiero brindarme ese instante de recogimiento, $y$, con una hon- 
da emoción que ha de recorrer el claustro porque no es mentida, quiero evocar la memoria de mi maestro el profesor CARva, I,O, de mi maestro el profesor Ondozor,a-para qué decir más?-las más altas cumbes en la historia del profesorado, de la cirugía y de la medicina del Perú, y otrecer el ejemplo de esas vidas a la consideración de todos como un ideal nuestro, de valores auténticos en el profesorado nacional.

En estit añoranza del pasado que me pertenece al presentarme por tercerá vez ante la consideración de la Facultad partá sostener un programa de concurso, viene necesariamente a mi espiritu el recuerdo de otras horas coino ésta, vividas en esta misma tribuna y que se fueron, cons sus inquietudes y amarguras, dejando lozana una flor de ilusión que no se ha marchitado porque en ella puse lo mejor y más honrar\}o de mi vida. Séame, pues, permitido, para justificar mi presencia en este sitio, repetir las palabras que pronunciara aquí mismo, hace yá diez años: hay que trabajar.y esperar! Desiués de todo-dice el protesor Richris-se llega o no se llega. No importa: se ha trabajalo, se ha esperado. Tal es ubjeto de la vida: trabajar cona uma esperanza que brilia delante de nosotros como una extrella. A ustecles toca decir si el opositor de hoy ha permanecilo ficl a sus convicciones de hace ya drez años. Fu cuanto a mí. debo declarar que tal es el credo de los mios: trabaiar y esperar, y tal también mi profesión de fe en la sartera del profesorado: trabajar y esperar! Por eso estoy aquí nuevamente, clespués de diez años, ahondanclo el surco por tercera vez, señores Catedráticos.

Amor al pasadio que constituye la nacionalidad cuando vincula al momento actual por un proceso ideológico de mejoramiento-hace cien años Unanub, ayer Ca RVAL1,o y ODRioZOLA, hoy DAMMERT-y hablo de él porque se fué. Trabajar, esperar y seguir imperturbablemente adelante pese al la gritería de los que se sienten estorbados que más taruehan de callar y aplaudir. Gratitud paral los que nos dieron en la vida su sonrisa comprensiva; pala mi maestro el profesor AvendaNo que me abrio las puertas de la enseñanza, para ustedes señores Catedráticos y para ustedes señores estudiantes que, en momentos agitados de la vida universitaria-que ya no volverán porque la inquietud no es ya solanente estudiantil sino de todo el claustro-confiaron en mi esfuerzo. $Y$ ya que hablo de esfuerzo, que haya respeto y tolerancia: que haya respeto porque lo merecen los que aquí arriba hacen el 
sacrificio de su esfuerzo. Que haya toierancia pala los que alláabajo recuben el frutode ese esfuctzo. Que la verdad remplace al cror alli donde se eilcuentre. Que el gesto traduzca loque está en la conciencia. Tales son los mandamientos de esa religión de amor, de trabajo y de verdad, que es el espiritu del protesorado del pás. (A plansos.)

\section{II.-Bases y finalidad de la Patologia Interna}

La Patologia Interna asienta hondamente sus raices en las ciencias funclamentales de los estudios médicos, en cuyo inagotable acero busca sus interpretaciones, y florece en armonía con las doctrinas médicas de la épocá en que vive. Por eso fué humorística con Hipocratess, luego organicista. con Virchow, quizo ser más tarte cxclusivamente infecciosa con Pastrizk y es ahora fisiológica con Grassrer y los médicos contemporáneos. Se nutre de todas ellas y recoge de la Semeiologia General los elementos esenciales para el establecimiento de las entidades nosográficas. Mientras que la Clínica estudial al enfermo, la Pa tología estudia la enfermedad que en buenas cuentás no es sino una abstacción que la razón háce posible. Lál Patulogía esturliác entidades de razón y para ellon el espiritu ha de buscar, donde la encuentre, la interpretáción más racional de los cuadros morbosos. Por eso acule a la Anatomia cuándo se tratil de un diag-

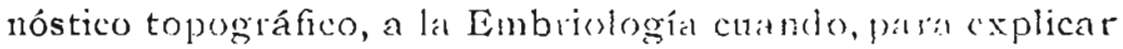
los casos de macrosenitosomia, de seudo-hermatiodismo adquirido e hirsutismo, en los cásos de neoplasias de las cápsulas supratrenales o sindrome de Garrars, recherda que anduvieron escondidos juntos en el embrión los blastemos que más tarde habrían de diferenciarse en cápsulas supra-renales y testículos u ovarios, conservando, no obstante su desigualdad anatomica, estrechas correlaciones funcionales, que una perturbación patológica podría, en algún momento, poner de manifiesto; a la Filogenia cuando se detiene a considerar la frecuencia de los cánceres en los órganos nuevos y su rareza en los de estirpe más antigua, como ocurre con la diaria realidad de las neoplasias del estómago y del recto. en contraposición a los excepcionales cánceres del intestino: los primeros, órganos derivados del intestino primitivo, mientras que el segundo, solo prolongación del mismo; a la Quimica, cuando en presencia de sindromes, que los ciruja: 
nos mejor que los médicos conocen, de aparente cistitis con disuria, orinas amoniacales, dolores lumbares y af́n cólicos nefríticos, pero en los que la infección no se encuentra, recuerda, como brillantemente lo ha demostrado el profesor GRASSET hace apenas algunos meses y nosotros hemos tenido ocasión de comprobarlo, que hay una cliátesis alcalina que cede, por lo menos temporalmente, al empleo adecuado desubstancias que mantengan la acidez de los humores y que exige un cambio radicall en lo que a dietética se refiere, proscribiendo la rutinaria costumbre de los régimenes sir atbrmina; a la Física cuando, para explicar los fenómenos anafilácticos, rompe la tradición de las intoxicaciones para crear un capítulo nuevo de equilibrio coloidal como lo ha hecho ver la Escuelá de WIDAL, de Lumere y de Kow peschi; a la Auatomía Comparada cuando, para explicar los sindromes parkinsonianos y mioclónicos, recuerda al hombre de ciencia que los cuerpos estriados-núcleo cáudál y núcleo lenticular -que allá por las épocas en que andaba discurriendo por las aulas de estudiante, se me an tojaban colocados en el cerebro para complicar su estructura y mis estudios, por cuanto no les encontraba a plicacion-digo que, recuerda al hombre de ciencia que aparecen en la escala de los scres, finicamente cuando éstos pasan de la vida acuática a la terrestie y que por consiguiente, algun papel han de jugar en el mantenimiento y en el control de los novimientos automáticos. Efectivamente, mientras que la porción interna de a mbos núcleos-el palleo striatum-la más vieja, asegura el control automático de los movimientos ásociados que dan estabilidad al ser en la rida marítima, la porción externa-el neostriatum-cuyo desarrollo es mayor a medida que avanzamos en la escala de los seres, aparece como un freno cuando necesidades nuevas de la vida terrestre hacen inutil tal expendio de energía. Y he ahi porqué, como bien lo han demostrado los trabajos de Tilney y la Escuela Americána, en ciertos casos patológicos, roto un sistema, la influencia del otro se deja sentir y el parkinsonismo aparece, mientras que en otros se manifiesta la atetosis con su rico cortejo de movimientos reptiformes. Acude a la Histología Fisiológica cuando nos dice que en el estómago a vivel del cardiás y de la pequeña curvadura, en el duodeno, en el ángulo duodenoyeyunal, en la válvula ileo-cecal, en un punto medio del colou transverso a proximadamente y en itro punto del colon pelviano, existen elementos perfectamente bien diferenciados 
que tienen a la vez del sistema muscular y del nervioso $y$ que son al aparato digestivo lo que el haz de His es al corazón: los centros nodales estudiados por Kerter. Acude a la Anatomia Patológica cuando nos demuestra que las alteraciones de textura de esos centros explican esa paradoja de los espasmos y atonías de la enfermedad de Glenard. Acude a la Fisiología Patológicá con un criterio quirúrgico cuando nos dice que en las resecciones de las Giceras de la pequeña curvadura, las resecciones cuneiformes van a contribuir, -como lo há demostrado el fisiólogo americano Ar.varez, a la dila tación del estómago, puesto que ya no bay sincronismo de onda, toda vez que las que pásan por la pequeña curvadura se encuentran interrumpidas. La cirugía anatómica creb una arritmiá estomacal. Acude a la Microbiología en esa gama infinita de las enfermedades infecciosas. Acude a la Psiquiatría cuando nos da la clave de ciertas enfermedades aparentes que ocultan los tormentos del espíritu; a la Psicologíc cuando nos dice que el médico no debe separarse del lecho del enfermo sin dejar por lo menos una palibra de consuelo. Acude a la Patología General que le da sus orientaciones. Acude a la Semeiología que le da su técnica. Acude. a la Fisiología en todos los casos, desde que la medicina de hoy es estrictamente fisiológica.

Por consiguiente, señores, si esto es así, el alumno al penetrar en lás aulas de lá Facultàd de Medicina, y con mayor razón al iniciarse en el curso de Patología Interna, debe conocer la importancia de estas cosas, que parecen de cierta aridez cuando se estudian aisladamente, pero que adquieren una floración exhuberante cuando se aplican al estudio de la Patología Interna, si ésta no ha de ser una mera repetición de cosás sino el taller donde se fragüe el pensamiento médico del alumno. (Aplausos.)

He aquî la doctrina de mi curso: romper con la tradición que quiere aherrojar al espiritu en la memoria; dar sueltá al alma y que ésta elabore con toda su capacidad razonadola el pensamiento del alumno. 


\section{III.-Criterio con que ha de estudiarse la Patología Interna}

¿Con qué criterio debe estudiarse la Patología Interna? Digo, señores, en mi programa que para que la enseñanza de una disciplina médica o de una ciencia cualquiera tenga sueficiencia máxima es absolutamente indispensable que responda a estos dos postulados: primero, que la doctrina del curso esté acorde con el pensamiento científico del momento en que se vive; $y$, segundo, que la enseñanza se conduzcal en armonía con los métodos de la Pedagográa Médica moderna.

Me apresuro a declarar que el criterio que va a inspirar la enseñanza del curso de Patología Interna es un criterio dináruico, funcional, fisiológico.

Señores:

Dice el profesor MaCklinzie que la enfermedad pasa por cuátro estados: un primer estado-formativo, diríat yo-de predisposición en que el individuo lo rnico que tiene es la vulnerabilidad hereditaria o adquirida; un segundo período, de lesión anatómica mínima, en que las perturbaciones son puramente fisiologicas, puramenr? subjetivas; un tercer período, de lesiones anatómicás considerables, tanto que ha sido fácil correlacionatas con el aspecto clínico del enfermo; $y$, por filimo, un cuarto período, de grandes lesiones anátomo-patológicas que se encuentran en lá sála de autopsias.

Pues bien; es curioso observar-dice Palerson, wno de los mejores educadores americános-cómo la merlicina de hoy ha acumulado una contidad considerable de hechos respecto de estos dos Gltimos estados, mientras que apenas si tenemos datos sobre el segundo periodo, en que las perturbaciones son subjetivas, y menos todaría sobre el primer estadode predisposicióv. Y MACKENziE, el insigue pensadoringlés, criticando duramen te la educación médicá en Ingla terra, escriba estas palabras: "Tenemos magníficos institutos de Anatomía Patológica destinados al estudio de las enfermedades después que el indivicluo ha muerto". Es preciso, paes, reaccionar y dirigir todas nuestras actividades del lado det segundo y primér periodo de la entermedad. Pues bien, señores, Mackenzie a firma que solo es con un critiero dinámico. 
fisiopatologico, funcional, como puede llegarse a la adquisicibn de la verdad referente a estos dos períodos de la enfermedad.

Con él vamos a incursionar en los distintos capitulos de la Nosografia.

En efecto, si comenzamos porla vida visceral, debe recordarse que toda ella está bajo la dependencia de un sistemá nervioso que la rige: el sistemá nervioso vegetativo; y si bien no es verdad, como lopretendieron los admirables creadores de la doctrina nueva En ERGER y Hiss, que de lat hipertonía de uno de estos sistemas dependen determinados trastornos y que de la hipertonía del otro dependen otros tipos clínicos de sentido contrario; o en otros térnmons: si ya no puede admitirse gur vago y simpatico representan formaciones nerviosas antitésicas, en cambio debe aceptarse - como lo asegura Canvon y la clínica lo demuestra-que la vida normal visceral es función del equilibrio orclenador $y$ regulador del sistema nervioso vegetativo, y que su desequi. librio produce cuadros que se mánificstan por fenómenos distuncionales de toda variedad, independientes o asociados, de los dos sistemas. En efecto, hace ya al reledor de tres años que he tenido ocasión de demostrar en la tesis de bachillerato de uno de mis alumnos, el doctor GuzMás DarRon, que en ciertrsé casos de pertubaciones puramente funcionales del estómago me ha sido dable encontrar alteraciones del quimismo gástrico que lá grálíca registró con movimientog de oscilación que trad acen fenómenos de hipertonía e hipotonia del mismo sistema; y lo que es más inportante, he tenido ocasión de presentár álimamente a la "Sociedád $\mathrm{Pe}$ ruana para el Progreso de la Ciencia", una comunicación so. bre dos casos con pertuibacione's funcionaies del estomago que presentan unas veces historia quínica y clinica rle hiperclorhidria, y otras, años después, una historia química y clínica de hipoclorhidria. Solamente con un criterio dinámico, fisiopatológico, es como ha sido posilile llegar át su rálzonada interpretación.

De otrolado, señores, cuando en un punto cualquiera del organismo visceral hay una lesión, se verificán alli dos clases de tristornos: uno "in situ" y otro que va a traducirse sobre organos alejados, con la circunstancia curiosa que muchas veces estos altimos son los predominantes. He allí por que la apendicitis como la colecistitis se enmascaran en un cuadro de hiperclorhidria, todos sabemos que los tuber- 
culosos pulmonares incipientes en su período de latencia se presentan como dispépticos.

Más todavía: en este mismo orden de cosas, es posible, con un criterio fisiopatologico, explicar los trastornos a dis. tancia, tal como ocurre cuando las colecistitis dan, por via refleja, un cuadro de fibrilación auricular.

Como vale la pena justificar las a firmaciones con los hechos, veamos ahora si este criterio es fructífero en inducciones nosológicas.

Hay una enfermedad que se presenta en el $90 \%$ de los casos no tratados apropiadamente, enfermedad silenciosa que solamente-como dice BARKBR-se anuncia alclínico por sus complicaciones, es decir, cuando es casi irreparable; me refiero a la "aortitis sifilitica". Efectivamente, la Patología de hoy conoce la aortitis cuando el espiroquete se ha infiltrado en las paredes del vaso, ha comprometiojo los plexos y producido el drama de la angina de pecho, o cuando, en su afán por los vasos, ba producido la destrucción de su capa media y constituido un aneurisma; y bien sabemos cuán poco podemos hacer en contra de los áneurismas: o, en otros casos, cuando el espiroquete se fragua camino en el nacimiento de la aurta, hace perder a los vasos su elasticidad propia y crea una insuficiencia abrtica. Pero hay un primer periodo silencioso que no se revela por signos físicos, pero que puede allunciárse en ciertos casos por signos hisiopátológicos. Pues bien, en algunos de esos casos me ha sido posible encontrar una historia de dolor epigástrico tardío calmado por los alimentos y por los alcalinos; dolor a veces transfixante, sobreviniendo por periodos o crisis, con constipación, demostrando la hipertonía del vago gástrico, y en los cuales el examen radioscópico me ha permitido encontrar, con la mayor sorpresa, casos auténticos de aortitis en los cuales ha sido posible emplear una tera péutica específica. He aquí, pues, como es posible con un criterio f́siopatologico ir al encuentro de esos períodos silenciosos de aortitis que yo he descrito con el nombre de Aortitis entuascatada.

Sigamos, señores, con este criterio incursionando en otros campos de la Patología. Dice HaAd que toda vez que en el organismo visceral hay uua lesión, ésta es el punto de partida de un bombardeo de reflejos que llegan a determina. dos segmentos de la médula espinal cuya excitabilidad áumenta; y entonces, como consecuencia de ese aumento de ex- 
citabilidad, todas aquellas impresiones que llegan al mismo segmento procedentes de los 6rganos de relación en virtud de un principio de metamería que yo no voy a recordar aquí van a ser sentidas en una forma desusada: generalmente van a acusarse por fenómenos hiperestésicos. Así se explica peifectamente bien porqué en los casos de frlcera del estómago puede encontralse una zona hiperestésica correspondiente a la porción más anterior de inervación del $X$ nervio intercostal clerecho, y en los casos de fricera del duodeno en igual zona del lado izquierdo; en los caso de colecistitis, una zona hiperestésica a nivel ie la porción más anterior del IX nervio torácico derecho. En los casos de a pendicitis se sabe perfectámente que lá zona hiperestésica, corresponde a la porción más anterior de inerración del XII torácico derecho. He aili, pues, como con un criterio fisiopatológico podemos llegar al entendimiento de sintomas tan alejados de lesión.

P'ero hay más todavia. Voy a refarirme a los trabajos de Potringer. Y particularmente a la interpretacion-que en el fondo es el objeto de la Patología Interna-de lo que es el tórax alado del tuberculoso. El pulmón recibe inervación simpática de los ganglios correspondientes al $1^{\circ}, 2^{\circ}, 3^{\circ}, 4^{\circ}$ y $5^{\circ}$ pares torácicos. No está demostrado, pero hay una tendencia grande a aceptax que está inervación puede ser támbién conductora de los estimulos que parten del brgano en. fermo. En estas condiciones, pues, si suponemos que existe una lesión tuberculosa, va a ser ésta el origen de un bombardeo de reflejos que llegarán á la médula por estas mismas vías. I, uego, por medio de nemonas conectoras, van a ponerse en relación con los nervios cervicales que van a los músculos $y$ piel del cuello. Esta inervación hechat por los nervioscervicales y particularmente por los pares $3^{\circ}$ y $4^{\circ}$, corresponde a a los músculos: esternn-cleido-mastoideo, escalenos, pectorales, diafragma, trapecio, elevador del ángulo del omb plato y romboides. De otro lado, esta misma inervación va a la piel correspondientes a la misma zona metaméricá. Ahora bien, se explican así perfectamente los hechos siguientes: Toda rez que haya una lesión del pulmón que tienda a la cronicidad, será el punto de particla de reflejos víscero-sensitivos, viscero-tróficos y víscero-motores: víscero-sensitivos que se van a manifestar en zonas dolorosas de la fosa supra-espinosa; víscero-motores, que se van a revelar por la fijeza del diafragma del lado afectado o por el compromiso de los 
otros músculos que corresponden a la misma inervacion, por Gltimo, reflejos visceros-tróficos, cuando la lesión en los mísculos citados tiende a una mayor cronicidad, que a la larga producirán lo que es el tórax alado del tuberculoso. Pen. sando con un criterio fisiopatologico es como ha sido posible llegar a la demostración de está verdád: que el tómax alado del tuberculoso es funcion de reflejos, cuyo punto de partida se encuentra en el pulmón y que se expresan a través del sistema nervioso de la vida de relación.

\section{Señores:}

Yo no creo que aquí, dada lá cultura de las personas que me escuchan, puliera encontrarse un espiritu exigente que dijera este criterio está muy bueno, corresponde evidentemente a estos casos; pero cimo aplicarlo a las entermedades infeciosas?; cómo aphicarlo a perturbaciones puanmente funcionales como la neurosis? A tal persona yo le áconsejuria que leyese los capitulos pertinentes de la parte razomala de mi programa. Përo de todas maneras, guicre acceder a esta invitación que yo mismo me hago. Todos los vasos del ahdomen inclusive los vasos portar reciben inervación le los esplácnicos. Elábdomendice LaNGI.ON BRown-es wn gran le:ho sanguinto que, en virtud de un refiejo fisiológico. juega papel impertance en ia circulación, nantenicnio normal la tensión arterial. Por eso se explicar que tola vaz que haly femómenos de raso-dilatación de la piel, apracen iumediatamente fenómenos vasoconstrictivos del abelomen. Asegura Rosa, en su reciente taibajosol)te fisio-palologia quirargica, que cuanto hay una lesión intecciosa del peritonco, lo primero que ocurte es una vaso-diatacion, y conforme la peritonitis aranza, la vaso-dilatación abdominal avanzad igualmente; con esta cir. cunstancia, que no hay relacion directa entre la extensión de la infección peritoneal y los éminenos vasodilatadores, sino que, en un ginn numero de veces, basta una lesión minima para que se opere una parálisis vasomotora formidable. Entonces se explica porque en los casos de abdomen agudo hay frecuencia y peciueñez del pulso, caída euorme de la tensión arterial, cianosis, colapso, etc., puesto que el individuo-pen. sando con un criterio fisio-patológico-se ha sangrado dentro de su propio abdomen.

$Y$ en lo que se refiere, ahora, a las neurosis viscerales, ¿cómo a plicar este criterio? Asegura DakWIN, en su"Trata- 
do sobre las emociones del hombre y de los animales", que "En la agonía del dolor casi todos los músculos entrán en acción..... porque ha sido el dolor a través de incontables generaciones el acica te de los esfuerzos más variados y diversos para escapar de las causas de sufrimiento..... Un hombre $\leftrightarrow$ un animal que quiere huir por el terror de la desesperación es enormemente peligroso". "El hombre a través del tiempo ha aprendido a esca par del peligro corriendo aceleradaumente o por medio de una violenta lucha, $y$ tales esfuerzos obligan a latir más rápiclamente el corazón, a acelerar los morimientos respiratorios, a acrecentar el pecho, a dilatar las marinas". X CRILE describe los mismos hechos en un moderno simil. Dice asi: "El mismo hombre, hoy, desde su escritorio, bajo cuyo comando se moviliza la complicada máquina de la civilizacion, frente al terror de una catástrofe econamica manifiesta su miedo en los mismos términos que en la lucha fisica ancestral. No puede tene: sblo un miedo intelectual; no puede temer desal pasionadamente; sufre con sus mismos organos, los que están estimulados o inhibidios come en las luchas de la edad de piedra..... La naturaleza sobo tiene una clase de medios para responder a la emoción y cralquiera que seà la cálusá, se produce en términos iclénticos". Y en efecto, como lo ha demostrado Cannon y su Escuela, la representación orgánica de la emoción está dada por fenómenos de hipertonía del simpático, a saber: táquicardia, respiración trecuente, dilatación de la pupila, sequedau de la prel y de las mucosas, angustia precordial, movilización del glucógeno para subvenir a las necesidades de la lucha, etc. He aquí. pues, como hasta en el mecánismo de la emucion es posible encontrar la huella fisiológica. Y se comprende entonces-para no háblar más sobre el particu. lar-cómolas desviaciones de ese dinamismo nervioso pueden ocasionar perturbaciones funcionales toda vez que lleguen a la conciencia actos que normalmente se esconden en la actividad del sistema vegretatlvo. Por eso durante la última guerral curopea, después de los rálids aéreos, aumentaron los casos de cliabetes y de mal de BASEduw; y por eso, también, toda vez que por motivos de inquietud espiritual los actos que andan normalmente ocultos en el sistema vegetativo, suben a la conciencia, se originan neurosis viscerales.

De otro lado, en lo que se refiere al primer periodo de la enfermedad de Mackenzir también se encuentra la huella fsiopatológica. Vamos a demostrarlo: 
El profesor francés Mouriqaun asegura que el artritismo es una palabra en las aulas estudiantiles, porque es científico asegurario así, pero que luego en la práctica diaria nos tropezamos con él a cada instante. Vale, pues, la pena buscarle explicacion. Vamos, con un criterio fisiopatológi$c o$, a indagar lo que esté a nuestro alcance o a teorizar sobre el concepto científico del artritismo en el momento actual. En él están comprendidos: gota, obesidad, diabetes, choques anaffácticos, asmá, litiasis renal, jaquecas, litiasis biliar, etc.

La obesidad se encuentra caracterizada por una ley bioquímica: la hipoglucemia; la diabetes que está en el polo opuesto y que muchas veces es el término obligado de la obesidad, lo está por otra: la hiperglucemia (no hay diabetes sin hiperglucemia; cuando ésta no existe, seguramente es de causa renal). La litiasis renal se caracteriza por una ley bioquímica: la hiper-uricemia, la hiper-oxilemia o la alcalosis; fuera de estos casos no hay litiasis renal a no ser que se llame así a los cálculos que se producen por infecciones ascendentes. El reumátismo diatésico está caracterizado por una ley bio-química: la hiper-uricemia. La litiasis biliar se caracteriza por una ley bio-química: la hiper-colesterinemia. El asma, por procesos bio-químicos; el choque hemoclásico, por la caída de la tensión arterial, la inversión cle la fórmu. la, etc. Perósta, señores, es una taz de la interpretación. Vamos ahora, con un criterio fisio-patológico, a incursionar dentro de la Patología para ver que se encuentra en el fondo de estas perturbaciones. Hablo esquemáticamente. En ciertas lesiones de la hipofisisis se presentan cuadros de adiposis perfectamente características con hipo-glucemial. El cstado de insuficiencia tiroidea se caracteriza en ocasiones por un cuadro de adiposis con hipo-glucemia. En la obesidad que precede a la diabetes existe una asimilación superior de los carbohidratos a la que constituye la asimilación normal; de manera que cabe decir que algun papel debe ju. gar la insulina y el pancreas en la obesidad que no está filiado por el momento. De otro lado, otras lesiones de la hipó. fisis producen acromegálicos, hiperglucémicos y glucosúricos. En el mal de Basedow hay crisis de hiper-glucemia en que aparece azGcar en la orina. Es in util hablar de las lesio. nes del pancreas que próducen el cuadro de diabetes.

Pero hay mas todavía. Hay ciertos casos de reumatismo diatésico de los 40 años, en sujetos obesos, mejor dicho 
en enfermos obesos, con perturbaciones especiales del trofismo de la piel y de las uñas, con una frigidez especial, sensación de frío intenso, constipacion inveterada, todo lo cual ha indicaldo que existen alli evidentemente perturbaciones de orden hipo-tiroideo. Y entonces se explica porqué ciertos de esos cuadros de reumatismo diatésico hipo-tiroideo se benefician con la administración de glándula tiroides, combinada a lá terapéutica apropiada. Por último, todos sábemos có. mo en la crisis coloidoclásica y vagotónica del asma, la influencia de la adrenalina es decisiva, lo que hace suponer que algán papel desempeñan las suprarrenales en la producción de estos trástornos.

Por todo lo cual se infiere que nos hemos alejado de las constantes bio-químicas para entrar en un terreno más pro-

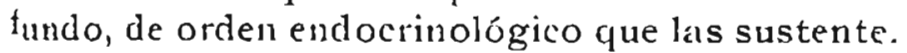

$\mathrm{Y}$ ahora bien, los obesos, los diábéticos, etc., etc., los ar. tríticos en general son aquellos que nacen con estigmas característicos que el clínico está aprendiendo a conocer y buscar. En este primer período de la entermedad de Mackenzis: en el período constitucional. De artríticos proceden los niños hermosos, obesos, semejantes a las criaturas de los cuadros de Rubens; son esas las criaturas predispuestas at las enteritis, al eczema, a la espasmofilia; son los que tienen ganglios grandes, timo grande y que mueren slibitamente. Son éstos los sujetosáplásicos arterialesque más tarde van a hacer las hemorragias cerebrales de los 30 años y a presentar todas las manifestaciones del artritismo. Por consiguiente pues, ya se ve c 6 mo en el fondode estas perturbaciones se encuentra el estado timo-linfático de Paltauf, la diatesis exudativa de Czerny. Y como si esto no fuera bastante, quizás si en el fondo de estas diátesis hay una herencia sifilitica o tuberculosa.

Se me dirá, señores, que estas son hipotesis; pero es que sobre hipótesis se levantan todas las ciencias; sobre hipotesis se hace la vida diaria de la humanidad, y si sobre hipbtesis hemos de edificar la explicacion razonada de los bechos, ibenditas sean las hipótesis! (Aplausos.)

Después de esta incursión extensa y honda en todos los campos de la Patología, en todos los periodos de la enferme. dad, me parece haber fundamentado la necesidad del criterio fisiológico en el estudio de la Medicina. 


\section{IV.-Cómo debe enseñarse la Patología Interina}

Me es verdaderamente sensible molestar la atencion de los señors Catedráticos, repitiendo frases que pronuncié aquí mismo hace diez años; pero como tengo que ser consecuente con mis ideas pedagógicas, es preciso que insista en ellas. Decía en ese en tonces y repi to a hora, que la enseñanza no puede sernisolamente teóricá ni exclusivamente práctica; que la enseñanza debe ser teórica y práctica al nismo tiempo o deja de ser enseñanza. Protestaba de la enseñanzá puramente té6. rica, con más razón ahora, en el curso de Patología interna, en que una feliz disertación oratoria puede llevar al espiritu del alumno el conocimiento de una entidad de rázón, y entoncés éste se imagina que ya no habrán dificultades cuando se tropiece con la realidad de los entermos. Sinembargo, senores, uada más erróneo. Me elevaba támbién contra la enseñanzá prácticá que no iba a sacarde la Facultad profesionales sino empíricos, y ahora, como entonces, creo que lo que consulta mejor los intereses de la docenciá mélica es el concepto integral de la enseñanza: que éste sea a la vez teórica y práctica. Por eso creo que debe arrancar desde el entermo mismo; porque si el alumno objetiva su ense ñanzacon un "câso"; si tropiezá con las dificultades de interpretación de ese caso y si a su lado el profesor enmienda erroles y rectifica opiniones, llegárá fácilmente a la edificación de una: verdad que ya no ha de olvidar más, porque en ella han intervenido vatios factores: el enfermo, el alumno desempeñando papel activo en el trabajo y el profesor como guía es. piritual en todo instante. Ya Trousseau decía bace machos años, en una ambición de mejoramiento de la enseñan. za médica, que el alumno cuando estudia la Patología Interná se cree genio; en cambio cuando entra en la clínica siente que la tierrá se abre bajo sus pies. Y Dieurafoy, uno de los hombres más ilustres de la medicina francesa, aseguraba que clínicá y patología no son cosas distintas sino que son ciencias que en todo momento se dan la mano y se hacen mutuos préstamos y concesiones. Decía-de esto hace más de 25 años-que el profesor de clinica hace patología cuando para hacer el diagnóstico tiene que recurrir a la No. sografia y referirse a la enfermedad; y que el profesor de patologia hace clínica cuando al describir la enfermedad tiepe que referirse necesariamente a la historia de un enfermo. Y 


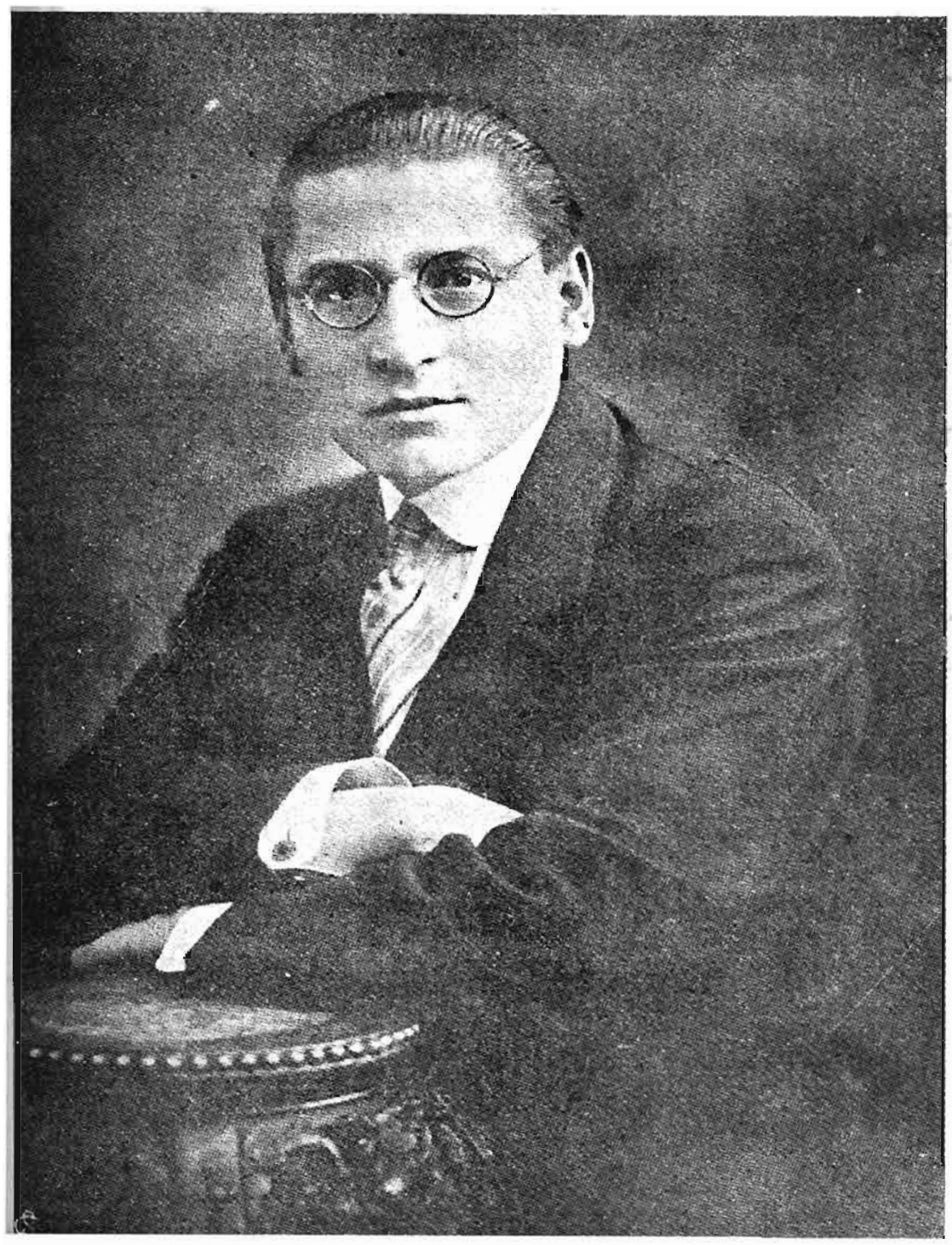

Profesor Doctok Carlos MONCE Y M CateUR.TICO l'KINCIPAT. 1) I: Nusogralía Mŕdica 
luego agregaba: "Yo no concibo cómo puede ser alguien pro. fesor de Patolugín Interna sin ser al mismo tiempo médico de hospital".

Los señores Catedráticos me excusarán que, como una prueba de que la enseñanza debe ser tebrica y práctica, me refiera a dos casos que he vivido. El primero en el año de 1912, en la Escuela de Medicina Tropical de Londres. Se trataba de un enfermo que se presentó con una lesión botonosa de la cara, ulcerada, ligeramente ro. ja en los bordes, con una exudación que se había concretado en costras y que sangraba fácilmente. El protesor Manson presentó el caso y áseguró que era un ejemplo tipico de leishmaniasis de la piel y luego le pregunt6 su procedencia. - "Vengo del Tambopata"-respondio el enfermoEntonces me di cuenta de que yo había visto casos idénticos y de que la "uta", "cquecpo", "tiac"-"araña", etc., no eran sino leishmaniasis de la piel, como pude comprobarlo fácilmente años después en una excursión a la zona montanosa del departamento del Cuzco.

Elotro caso es éste: En el año de 1917, en un viaje que hacia del extanjero al pais, aprendi que habia una enfermedad nueva caracterizada por tres signos: fiebre de tipo irregular, somnolencia y parálisis de los pares craneales. El concepto se gravo en mi espíritucomo un hecho puramente teorico y alllegar a lima tuve la suerte de describir el primer caso de Encefalitis epidémica.

Como se hal discuticlo un poco respecto de la enseñanza práclica en esita cáledra, se me permitirá que cite referencias de auturiclacles médicas y docentes.

He aquí la opinión del profesor Devoyas, director honorario de la Escuela de Medicina de Dijon: "Es en el hospital, y arsi exclusivamente alli, que el estudiante adquiere lo que será más tarcle, durante toda su vida profesional. El hospital es para el estudiante de medicina, lo que el taller del pintores para sus alumnos. No es ni escuchando al maestro discurrir sobre la pintura, ni aún viéndolo trabajar que el estudiante se forma, y si manejando él mismo el pincel bajo la rista y dirección del maestro. Esta superioridad de la enseñanza hospitalaria sobre la enseñanza puramente térica de la Patología dada en un anfiteatro, ha sido también reconocila. Hace 15 o 20 años que se resolvió en principio la transformación progresiva de las cátedras de Patología en chted ás de clinica, tal como ha ocurrido en la Facultad de 
Medicina de Estrasburgo, en que la enseñanza de la Patología estaba confiada al Catedrático de Clínica desde antes de la guerra".

Por su parte, el profesor Brunnon, Director de la Fácultad de Medicina de Rouen, dice así: "Actualmente nuestras. escuelas de medicina son ya centros de instrucción activos cuando tienen anexos grandes hospitales. Toda la cuestión está allí. La Escuela de Medicina es el hospital; la clínica es la medicina. Es en el hospital que se forma el merico clarovidente, discreto, escéptico (discretámente escéptico di. ria yo) es decir no peligroso".

El profesor LABBE de París, refiriéndose a la enseñanza: de la medicina en lá Argentina, dice lo siguiente: "Lá eriseñanzá de la Patología Interna y de la Patología Externa se hace generalmente en el hospital. El profesor titular y los adjuntos hacen un curso regular con programa determinado que ellos ilustran, cada vaz que ésto es posible, con la presentación de enfermos de su servicio. Yo he encontrado esta misma tendencia en el Brasil, donde el profesor de pátología sueña con anexar a su instituto un servicio de medicina, a fin de enseñar las aplicaciones inmediatas de la patología a la práctica médica. Es ésta una feliz concepción que párece no sufrir en la Argentina ninguna dificultád práctica."

En la universidad deJohn Hopkin, he aqui como describe el profesor BenolT el sistema de euseñanza: "Supongamos que se trate de enfermos del pulmón. El profesor preguntá al es. tudiante si ha visto casos de neumonia y cuáles son los signos que presentaban los enfermos, cómo ha examinado los pulmones enfermos, qué signos físicos ha encontrado, cuáles son los que se encuentran generalmente, qué enseñanzas de control ha podido obtener por los exámenes de laboratorio. Y cuan. do ha terminado estas preguntas agrega: ¿Ha leido usted el capítulo de neumonia? Que es la neumonia, cuáles son sus causas, y así sucesivamente. Los errores son corregidos, los puntos oscuros se aclaran y el alumno sale de esta conversación perfectámente documentado. Está recitación tiene la ventaja de ser algo vivido que excluye por completo la monotonía y la fatiga a condición de que el alumno tenga el sen. timiento de su propia respunsabilidad al cual debe hacer ho. nor. Los americanos pretenden que esta manera de comprender la enseñanza médica da resultados mucho más satisfactorios que los métodos seguidos generalmente."

Por altimo, segán el profesor Ren6n, actual Cátedrá. 
tico de Patología Interna de la Facultad de Medizina de París, se debe enseñar la Patología Interna de una manera tan práctica como sea posible, pero sin descuidar las consideraciones generales capaces de desarrollar en el alumno la observación, la reflexión y el juicio indispensables a un médico; más todavía, va hasta el punto de haber hecho semanalmente una lección de semeiología técnica y crítica, que yo agregaria debe ser interpretativa.

\section{Señores:}

He llegado al término de mi discurso. Creo que el profesor ha de ser un guia espiritual para el alumno; creo que lo que hay que dar a éste es el concepto integral, la capacidad razonadora; creo que el alumno debe estar familiarizado con los hechos, pero támbién con las verdades cientificas. Creo, como en la república de Pla'ton, que es preciso dar buenos médicos al estado, buenos médicos familiarizados con un gran numero de enfermos y sobre todo con un gran nímero de sanos, porque no hay que olvidar que el hombre esconde los tormentos de su espíritu con el disfraz inconsciente de una enfermedad aparente, las neurosis viscerales; y de alli porqué el Quijote resulta muchas veces mejor que los libros de medicina, porque la cultura general vale tanto o más que la cultura médica.

Creo que enseñar es antes que nada, como dice el profesor WIDAL, elegir y simplificar; y que a esta elección, a esta simplificación solo se llega cuándo se siente la sana vocación de la enseñanza, cuando se está profundamente penetrado de los límites de una ciencia, cuando se experimenta el placer de trasmitir el pensamiento de uno al alma del que se inicia.

Enseñar, señores, es en realidad hacer pensar a los alumnos. (Aplausos.) 